\title{
Aumento da Sobrevivência de Aedes (Stegomyia) aegypti (Linnaeus), em Condições de Laboratório, pela Ingestão de Néctar Extrafloral de Euphorbia milii Des Moul. (Euphorbiaceae)
}

\author{
Nathalia Cavichiolli de Oliveira ${ }^{\bowtie}$ Antonio Pancracio de Souza
}

Universidade Federal de Mato Grosso do Sul, e-mail: nathaliacavichiolli@hotmail.com (Autor para correspondência ${ }^{\bowtie}$ ), antonio.souza@ufms.br.

\section{EntomoBrasilis 7 (1): 48-51 (2014)}

\begin{abstract}
Resumo. O objetivo deste estudo foi determinar em laboratório se Aedes (Stegomyia) aegypti (L.), importante vetor da dengue e febre amarela, alimenta-se do néctar de Euphorbia milii Des Moul (Euphorbiaceae), planta comumente utilizada nos domicílios como cerca viva, e avaliar o efeito desta alimentação em sua sobrevivência. O tempo de vida de ambos os sexos foi acompanhado diariamente e um teste de detecção de frutose foi utilizado para a comprovação da ingestão de açúcar pelos mosquitos. O acesso diário ao néctar conferiu um aumento significativo no tempo de vida de machos e fêmeas (12,8 e 18,4 dias, respectivamente), em relação aos mosquitos mantidos somente com água (6,4 e 7,4 dias, respectivamente). Plantas em ambientes domésticos, produtoras de néctar e adequadas à alimentação do mesmo pelos mosquitos, assim como E. milii, possuem o potencial de desempenhar um significativo papel no orçamento energético de mosquitos. Um aumento na sobrevivência de fêmeas pode significar um aumento na probabilidade de infecção e transmissão de agentes patogênicos e nos machos um aumento na probabilidade de inseminação das fêmeas. Ainda que frequentemente desconsiderada em pesquisas ou táticas de controle, a propensão do mosquito Ae. aegypti em ingerir açúcares pode ser uma variável que confira vantagens a este vetor.
\end{abstract}

Palavras-chave: Mosquito; Ecologia de vetores; Néctar.

\section{Increases Survival of Aedes (Stegomyia) aegypti (Linnaeus) Under Laboratory Condition by the Ingestion Extrafloral Nectar of Euphorbia milii Des Moul. (Euphorbiaceae)}

Abstract. The objective this study was to determine if in laboratory, Aedes (Stegomyia) aegypti (L.), an important vector of dengue and yellow fever, feeds on the nectar of Euphorbia milii Des Moul (Euphorbiaceae), plant commonly used in homes as a hedge, and evaluate the effect of feeding on survival. The lifetime of both sexes was checked daily and a test for fructose was used for verification of sugar intake by mosquitoes. The daily access to the nectar gave a significant increase in the lifetime of males and females (12.8 and 18.4 days, respectively) in relation to mosquitoes maintained only with water (6.4 and 7.4 days, respectively). Plants in domestic environments, producing nectar and suitable for feeding by mosquitoes of the same, as well as E. milii, have the potential to play a significant role in the energy budget of mosquitoes. An increase in the survival of females can mean an increased likelihood of infection and disease transmission in males and an increased likelihood of insemination of females. Although often overlooked in research and control tactics, the propensity of the mosquito Ae. aegypti ingesting sugars can be a variable that confers advantages to this vector.

Keywords: Mosquito; Nectar; Vector ecology.

$\mathbb{M}$ achos e fêmeas de mosquitos podem suprir suas necessidades energéticas e assim aumentar seu tempo de vida alimentando-se de líquidos açucarados excretados por insetos esternorrincos, néctar floral e extrafloral (Foster 1995; GARY \& Foster 2004; MÜller et al. 2011). Essa alimentação pode ser modulada pela disponibilidade de fontes de açúcar adequadas ao consumo dos mosquitos (MARTinEzIbARRA et al. 1997; SPEnCER et al. 2005; Müller et al. 2011). A identificação destas fontes além de ampliar o conhecimento da biologia destes insetos, mais especificamente de seus hábitos alimentares, pode colaborar em projetos de paisagismo urbano, evitando o uso de plantas que possam beneficiar a sobrevivência de mosquitos, principalmente de espécies vetores de doenças. Além de contribuir em intervenções de controle, utilizando estas fontes como base para a elaboração de atrativos sintéticos tóxicos a mosquitos (Schlein \& Müller 2008), ou como iscas, pulverizadas com inseticidas orais (MÜLLER \& SCHLEIN 2006; MULLER \& SCHLEIN 2008).

Certos tipos florais podem proporcionar vantagens para alimentação de mosquitos, devido a sua arquitetura. Flores com nectários, pequenas, aglomeradas e de estrutura aberta criam uma área de pouso que facilita a visibilidade e a alimentação, sendo, portanto mais susceptíveis a serem utilizadas pelos mosquitos como fontes de açúcar (Foster 1995). Euphorbia milii Des Moul. (Euphorbiaceae), popularmente conhecida como coroa de cristo, espinheiro ou bem-casados se adéqua aos padrões descritos. Sua Inflorescência é do tipo ciátio, de estrutura semelhante à de uma só flor com quatro nectários evidentes. Esta espécie pode ser frequentemente encontrada no peridomicilio, sendo utilizada como cerca viva (SOUZA \& LORENZI 2008).

Identificações diretas de espécies vegetais fontes de açúcar e seus efeitos sobre espécies de mosquitos vetores, já foram descritos anteriormente em outras localidades principalmente para Anopheles gambiae Giles (Impoinvil et al. 2004; MANDA et al. 2007; Goungna et al. 2010). Para Aedes (Stegomyia) aegypti (L.), vetor da dengue e febre amarela, MARTINEZ-IBARRa et al. (1997) e SPENCER et al. (2005) realizaram estudos indiretos por meio de mosquitos

Agências de Financiamento: FUNDECT e CNPq 
coletados em campo para posterior verificação de frutose. Em seus estudos sugeriram possíveis espécies fontes, sendo o gênero Euphorbia citado entre as plantas floridas identificadas na área avaliada. O presente estudo buscou, portanto, verificar em laboratório se Ae. aegypti utiliza-se do néctar de $E$. milii como alimento e avaliar o efeito desta alimentação na sobrevivência deste importante vetor.

\section{MATERIAL E MÉTODOS}

Criação dos mosquitos. Os ovos de Ae. aegypti, foram obtidos em uma criação mantida no Laboratório de Bioquímica na Universidade Federal do Mato Grosso do Sul desde 2009, sendo renovada periodicamente por ovos coletados em campo. Os adultos da colônia são mantidos em uma dieta de sacarose a 10\%, água e periódicas refeições de sangue por meio de hamsters (Mesocricetus auratus) (Protocolo $\mathrm{n}^{\circ} 236 / 2009$, aprovado pela Comissão de Ética no Uso de Animais/CEUA/UFMS). Para o experimento foram criadas 200 larvas no mesmo estágio, por bandeja de plástico (40 × $27 \times 8 \mathrm{~cm}$ ), contendo aproximadamente $1 \mathrm{~L}$ de água. Para a alimentação foi fornecida ração para peixes ornamentais (Alcon $\AA$ ) trituradas até atingirem o estágio de pupa. Logo após puparem, foram transferidas para cada gaiola de acrílico ( $30 \times 30 \times 30 \mathrm{~cm}), 15$ pupas macho e 15 pupas fêmeas, seguindo o método de sexagem descrito por Consoli \& LourençoDE-OliveIRA (1994). A sala de criação foi mantida com temperatura e umidade ambiente $\left(28,5 \pm 1,7^{\circ} \mathrm{C}\right.$ e $\left.66,6 \pm 13,1 \%\right)$ e fotoperíodo de 12 horas (Claro: Escuro)

Tratamento e controle. Para o fornecimento de néctar foram coletados diariamente ramos de inflorescências nos horários de maior incidência solar, período de maior produção de néctar em E. milii, sendo posteriormente ensacados para que houvesse acumulo de néctar. A seguir, os ramos foram colocados dentro de copos plásticos com água, para maior durabilidade destes. A quantidade de inflorescências em cada gaiola foi estimada com base no número de indivíduos presentes na gaiola, de modo a suprir todos os mosquitos. Além das inflorescências no grupo tratamento, fora fornecido um recipiente contendo água. Este fornecimento reduziu a probabilidade dos mosquitos ingerirem o néctar para a obtenção de água. Já no grupo controle, foi oferecido somente um recipiente com água.

Teste de Antrona. Para a comprovação da capacidade dos mosquitos em se alimentar do néctar em $E$. milii, foram preparadas paralelamente duas gaiolas com 40 mosquitos (20 machos e 20 fêmeas) cada. No terceiro dia após o aparecimento dos adultos, em uma das gaiolas foi oferecido água e na outra, néctar durante quatro horas. Após este período, 10 fêmeas e 10 machos de cada gaiola foram coletados e congelados para posterior teste de antrona (VAN HANDEL 1972). Este teste detecta frutose, que é encontrada em plantas e produtos vegetais. $\mathrm{O}$ reagente foi preparado despejando $190 \mathrm{~mL}$ de ácido sulfúrico concentrado (98\%) em 75 mL de água destilada (solução diluída de ácido sulfúrico) e misturada com $75 \mathrm{mg}$ de antrona com $50 \mathrm{~mL}$ de ácido sulfúrico diluído (reagente de frutose). Os mosquitos foram esmagados individualmente em tubos de ensaio contendo $1 \mathrm{~mL}$ de reagente de antrona e incubadas em temperatura ambiente durante 60 minutos. A mudança da cor amarelada para azul escuro durante a incubação foi o indício da presença de açúcares vegetais não digeridos.

Análises estatísticas. A sobrevivência foi registrada pela contagem diária de Ae. aegypti vivos e mortos, sendo os últimos removidos. Os dados foram analisados separadamente por sexo e de acordo com a dieta oferecida. Os experimentos foram replicados seis vezes e limitados ao período de 30 dias, porque mais de $90 \%$ dos mosquitos analisados haviam morrido até o termino deste período. Os resultados de todas as réplicas do tratamento e controle foram agrupados e analisados como dois conjuntos de dados. As análises de sobrevivência foram feitas através do teste Kaplan-Meier para obtenção de curvas de sobrevivência e estas comparadas através do teste Log-Rank. Os mosquitos que acidentalmente escaparam das gaiolas, morreram afogados ou esmagados, foram descartados. As análises foram realizadas pelo software PAST versão 2.10 $囚$ (HAMmER et al.2001) e pelo Microsoft Excel $\AA$.

\section{Resultados}

O resultado do teste para frutose foi considerado positivo tanto para machos como para as fêmeas. As soluções que continham mosquitos mantidos com acesso ao néctar tornaram-se azuladas em menos de 60 minutos, diferindo do controle que permaneceu com coloração inalterada.

Fêmeas alimentadas com néctar obtiveram maior sobrevivência comparando-se as fêmeas com acesso somente à água $(\mathrm{p}<0,001)$ (Tabela 1). O fornecimento de néctar também propiciou um aumento no tempo de vida dos machos $(\mathrm{p}<0,001)$, porém estes valores foram significativamente menores do que o tempo de vida das fêmeas nas duas dietas oferecidas. As curvas de sobrevivência de machos e fêmeas mantidos somente com água ou com água e néctar mostraram um comportamento similar (Figura 1).

Tabela 1 Tempo médio (dias) de sobrevivência dos adultos de Aedes aegypti, conforme a dieta e o sexo, em laboratório.

\begin{tabular}{lcccccc}
\hline \multirow{2}{*}{ Dieta } & \multicolumn{3}{c}{ Fêmeas } & \multicolumn{3}{c}{ Machos } \\
\cline { 2 - 7 } & Média & Mediana & \pm DP & Média & Mediana & \pm DP \\
\hline Néctar & 18,4 & 19 & $\pm 7,7$ & 12,8 & 10 & $\pm 5,8$ \\
Água & 7,4 & 8 & $\pm 1,3$ & 6,4 & 6 & $\pm 0,6$ \\
\hline
\end{tabular}

${ }^{*} \pm \mathrm{DP}=$ Desvio Padrão

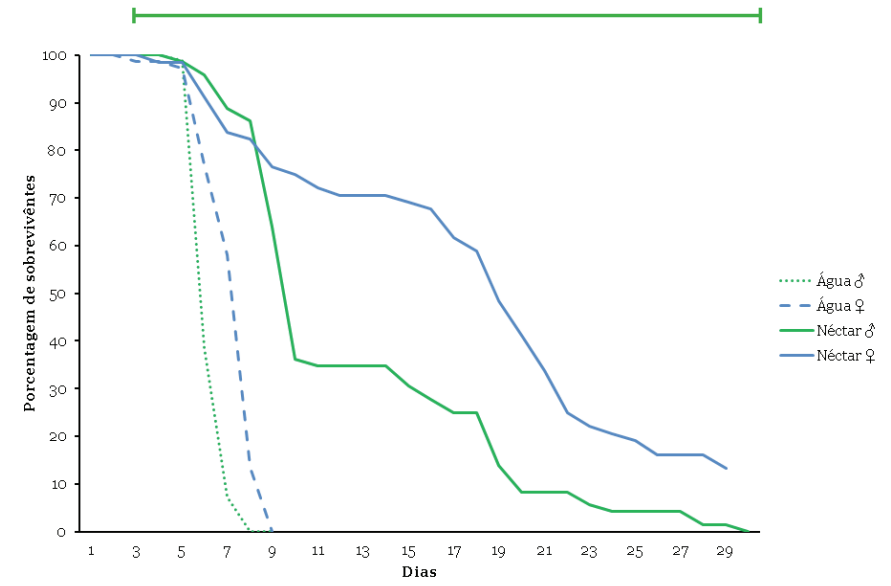

Figura 1. Curvas de sobrevivência de machos e fêmeas com acesso somente à água (controle) e néctar mais água (tratamento), em laboratório.

\section{DISCUSSÃO}

Machos e fêmeas de Ae. aegypti alimentaram-se do néctar secretado por E. milii e em ambos houve aumento na longevidade. Apesar de esta verificação ter sido feita em laboratório, é evidente o potencial que esta planta tem em conferir vantagens a este mosquito. O Ae. aegypti assim como E. milii, estão altamente associados aos seres humanos, vivendo nas proximidades de suas habitações. É possível que estes mosquitos obtenham açúcar a partir desta espécie vegetal e assim aumentem o seu tempo de vida. Nos machos, este aumento poderia significar maior probabilidade de inseminação das fêmeas e consequentemente maior número de descendentes (Foster \& Eischen 1987; STRAiF \& Beier 1996). Para as fêmeas, haveria um aumento no tempo de vida enquanto estas aguardam oportunidade de contato com humanos para refeições de sangue. O que elevaria assim o risco de infecção e transmissão de agentes patogênicos (JAHANGIR et al. 2008).

A eficácia do néctar de E. milii em manter a colônia de Ae. aegypti, a fácil visibilidade da presença ou ausência desta secreção e a associação desta espécie vegetal às cidades, ensejam a que se 
utilize esta planta em outros experimentos. Principalmente naqueles que tem por objetivo analisar os efeitos da alimentação de açúcar de origem natural sobre a biologia desse mosquito. No mesmo sentido está a grande maioria dos estudos que avaliaram os efeitos dessa alimentação sobre o Ae. aegypti, os quais foram levados a efeito com soluções açucaradas preparadas em laboratório (BRIEGEL et al. 2001; HARRINGTON et al. 2001; STRICKMAN 2006).

$\mathrm{Na}$ ausência de fontes disponíveis e adequadas ao consumo de líquidos açucarados, as fêmeas podem obter energia para suas necessidades vitais aumentando a frequência de seus repastos sanguíneos e consequentemente o contato com humanos (Foster \& Eischen 1987). Já os machos, que desempenham um papel essencial na manutenção da espécie, não possuem alternativa de alimento e tornam-se, portanto mais vulneráveis à disponibilidade de açúcar. Conforme demonstrado no presente trabalho, a ausência de açúcar pode acarretar desvantagens, sendo maior a mortalidade nos machos sem acesso ao néctar. GARY et al .(2009) sugeriu para An. gambiae, espécie com semelhanças ecológicas a Ae. aegypti, que o desempenho masculino está intimamente ligado às comunidades de plantas, pois em experimentos feitos em condições semi-naturais, sem açúcar, a capacidade de inseminação do sexo masculino era praticamente inexistente e STONE et al. (2009) semelhantemente demonstrou que na ausência de açúcar houve uma redução na sobrevivência e na capacidade de acasalamento, a ponto de levar o declínio da população.

Tendo em vista o comportamento alimentar de néctar e as vantagens por ele conferidas a machos e fêmeas de Ae. aegypti, era de se esperar um maior número de estudos que investiguem fontes de açúcares e suas relações com este importante vetor. Estratégias de controle poderão se beneficiar com o conhecimento dos diversos aspectos de sua biologia. O comportamento do Ae. aegypti em obter energia a partir de açúcares, pode ser uma variável, a qual mesmo não tendo sido muito considerada em pesquisas ou táticas de controle, pode estar influenciando na sua sobrevivência. Há uma infinidade de espécies vegetais e outras fontes de açúcares fornecidas até mesmo inconscientemente pelo próprio homem que necessitam ser investigadas como possíveis fontes alimentares de Ae. aegypti.

\section{AGRADECIMENTOS}

Os autores agradecem a Deus primeiramente, a FUNDECT/MS (Fundação de Apoio ao Desenvolvimento do Ensino, Ciência e Tecnologia do Estado de Mato Grosso do Sul) e ao CNPq (Conselho Nacional de Desenvolvimento Científico e Tecnológico) pelo financiamento da pesquisa.

\section{REFERÊNCIAS}

Briegel, H., I. Knüsel \& S.E. Timmermann, 2001. Aedes aegypti: Size, Reserves, Survival and flight Potential. Journal of Vector Ecology, 26: 21-31.

Consoli, R.A.G.B. \& R. Lourenço-de-Oliveira, 1994. Principais Mosquitos de Importância Sanitária no Brasil. Rio Janeiro, Fiocruz, 225p.

Foster W.A. \& F.A. Eischen, 1987. Frequency of Blood Feeding in Relation to Sugar Availability in Aedes aegypti and Anopheles quadrimaculatus (Diptera: Culicidae) Annals of The Entomological Society of America, 80: 103-108.

Foster, W.A., 1995. Mosquito Sugar Feeding and Reproductive Energetics. Annual Review of Entomology, 40: 443-474.

Gary, R.E. Jr. \& W.A. Foster, 2004. Anopheles gambiae Feeding and Survival on Honeydew and Extra-Floral Nectar of Peridomestic Plants. Medical and Veterinary Entomology, 18: 102-107.

Gary, R.E. Jr., W. Cannon III \& W.A. Foster, 2009. Effect of Sugar on Male Anopheles gambiae Giles (Diptera: Culicidae) Mating Performance, as Modified by Temperature, Space, and Body Size. Parasites Vectors, 2: 19.

Gouagna, L.C., R.S. Poueme, K.R. Dabire, J. Ouedraogo, D. Fontenille \& F. Simard, 2010. Patterns of Sugar Feeding and Host Plant Preferences in Adult Males of Anopheles gambiae (Diptera: Culicidae). Journal of Vector Ecology, 35: 267-276.

Hammer, O., D.A.T. Harper \& P.D. Rian, 2001. Past Palaeonthological Statistics Software Package for Education and Data Analysis. Disponível em: <http://folk.uio.no/ ohammer/past>. [Acesso em: 12/11/2012].

Harrington, L.C., J.D. Edman \& T. W. Scott, 2001. Why do female Aedes aegypti (Diptera: Culicidae) feed preferentially and frequently on human blood. Medical and Veterinary Entomology, 38: 411- 422.

Impoinvil D.E., Kongere J.O., Foster W.A., Njiru B.N., Killeen G.F., Githure J.I., Beier J.C., Hassanali A., Knols B.G. 2004. Feeding and survival of the malaria vector Anopheles gambiae on plants growing in Kenya. Medical and Veterinary Entomology, 18:108- 115.

Jahangir, K., C.Y. Lee \& J. Zairi, 2008. Effects of sugar and animal blood availability on attraction of Aedes spp. to humans. In: Proceedings of the 6th International Conference on Urban Pests (W.H. Robinson \& D. Bajomi, eds.). OOK-Press Kft, Budapest, Hungary. 197 - 201.

Manda H., L.C. Gouagna, E. Nyandat, E.W. Kabiru, R.R. Jackson, W.A. Foster, J.I. Githure, J.C. Beier \& A. Hassanali, 2007. Discriminative feeding behaviour of Anopheles gambiae s.s. on endemic plants in western Kenya. Medical and Veterinary Entomology, 21: 103-111.

Martinez-Ibarra, J.A., M.H. Rodriguez, J. I. Arredondo-Jimenez, B. Yuval, 1997. Influence of plant abundance on nectar feeding by Aedes aegypti (Diptera: Culicidae) in southern Mexico. Medical and Veterinary Entomology, 34: 589-593.

Müller G.C. \&Y. Schlein, 2006. Sugar questing mosquitoes in arid areas gather on scarce blossoms that can be used for control. International Journal for Parasitology 36: 1077-1080.

Müller G.C. \&Y. Schlein, 2008. Efficacy of toxic sugar baits against adult cistern-dwelling Anopheles claviger. Transactions of The Royal Society of Tropical Medicine and Hygiene. 102: 480-484.

Müller G.C., R.D. Xue \&Y. Schlein, 2011. Differential attraction of Aedes albopictus in the field to flowers, fruits and honeydew. Acta Tropica, 118: 45-49.

Schlein Y. \& G.C. Muller, 2008. An approach to mosquito control: Using the dominant attraction of flowering Tamarix jordanis trees against Culex pipiens. Medical and Veterinary Entomology, 45: 384-390.

Souza, V.C. \& H. Lorenzi, 2008. Botânica sistemática; guia ilustrado para identificação das famílias de fanerógamas nativas e exóticas no Brasil, baseado em APG 2. São Paulo, Nova Odessa, 704p.

Spencer, C.Y., T.H. Pendergast Iv \& L.C. Harrington, 2005. Fructose variation in the dengue vector, Aedes aegypti, during high and low transmission seasons in the Mae Sot region of Thailand. Journal of the American Mosquito Control Association, 21: 177-181.

Stone C.M., R.M. Taylor, B.D. Roitberg \& W.A. Foster, 2009. Sugar deprivation reduces insemination of Anopheles gambiae (Diptera: Culicidae), despite daily recruitment of adults, and predicts decline in model populations. Medical and Veterinary Entomology, 46: 27-37.

Straif, S.C. \& J.C. Beier, 1996. Effects of sugar availability on the blood-feeding behavior of Anopheles gambiae (Diptera: Culicidae). Journal of Medical Entomology, 33: 608-612.

Strickman D., 2006. Longevity of Aedes aegypti (Diptera: Culicidae) Compared in Cages and Field Under Ambient Conditions in Rural Thailand southeast Asian Journal of Tropical Medicine and Public Health, 37: 456-462.

Van Handel, E., 1972. The detection of nectar in mosquitoes. Mosquito News, 32: 458. 
Recebido em: 16/o1/2013

Aceito em: 21/01/2014

Como citar este artigo:

Oliveira, N.C. \& A.P. Souza, 2014. Aumento da Sobrevivência de Aedes (Stegomyia) aegypti (Linnaeus), em Condições de Laboratório, pela Ingestão de Néctar Extrafloral de Euphorbia milii Des Moul. EntomoBrasilis, 7 (1): 48-51.

Acessível em: doi:10.12741/ebrasilis.v7i1.305
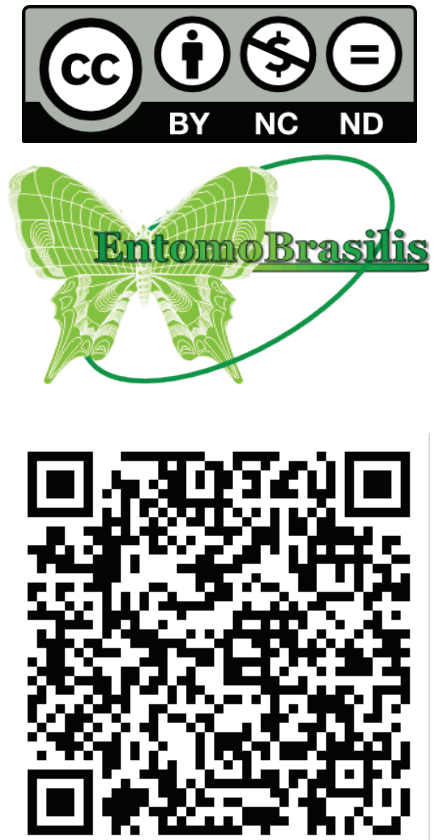